\title{
A Trade-Off Relation between Temporal and Spatial Averaging Scales on Future Precipitation Assessment
}

\author{
Kenshi HIBINO \\ Faculty of Life and Environmental Sciences, University of Tsukuba, Tsukuba, Japan \\ and \\ Izuru TAKAYABU
}

Meteorological Research Institute, Tsukuba, Japan

(Manuscript received 7 December 2014, in final form 25 September 2015)

\begin{abstract}
The detectability of climate change signals such as a precipitation change depends on temporal and spatial averaging scales. The present study aims to clarify the dependence of the detectability on the two averaging scales by analyzing the difference in daily precipitation between present (1979-2003) and future (2075-2099) climates. The dataset for the analysis is obtained from an atmospheric general circulation model. The robustness of the precipitation change signal is evaluated with the signal-to-noise ratio (SNR), which is often used in statistical tests to detect climate change signals. The SNR is increased and the detectability of the precipitation change signal is enhanced with increases in the two averaging scales. When either averaging scale is increased (decreased) with a constant SNR, the other averaging scale needs to be decreased (increased); this is the trade-off relation between the two averaging scales. The trade-off relation is obtained quantitatively and provides useful information for climate change impact assessments using various temporal and spatial scales or resolutions. The characteristics of the trade-off relation are found to differ qualitatively among the tropics, mid-latitudes, and subpolar regions and to derive from the precipitation power spectrum representing spatio-temporal scales of precipitation-related meteorological phenomena, e.g., baroclinic waves.
\end{abstract}

Keywords climate change assessment; atmospheric general circulation model; averaging scale; robustness of climate change signal

\section{Introduction}

Much research in climatology has assessed the impact of climate change from anthropogenic increases in carbon dioxide and other greenhouse gases in the earth's environment and human society.

Corresponding author: Kenshi Hibino, Faculty of Life and

Environmental Sciences, University of Tsukuba, 1-1-1

Tennoudai, Tsukuba, Ibaraki 305-8572, Japan

E-mail: hibino.kenshi.gn@u.tsukuba.ac.jp

(C)2016, Meteorological Society of Japan
Such research aims to detect climate change signals between present and future climates, as well as to quantify the magnitude of the impact. In the context of climate change assessments, the target periods or durations and spatial resolutions of the assessment outputs (not that of a numerical model) correspond to temporal and spatial averaging or smoothing scales, respectively. For example, if a climate change signal is obtained with temporal and spatial averaging scales $L_{t}$ and $L_{x y}$, the signal should be regarded as an averaged or representative signal of the domain and the 
period, not as a local and instantaneous signal.

The two averaging scales need to be determined with careful thought, because the robustness of the climate change signal depends on the averaging scales. Generally, global warming or annual-mean precipitation change is more easily detected than local warming or monthly-mean change, respectively. This is because the internal variability of the meteorological variables, which obscure the climate change signal, is reduced by the averaging operation.

The effect of the temporal averaging operation has been investigated by analyzing the results of general circulation models (GCMs) (Leith 1973; Chervin et al. 1974; Chervin and Schneider 1976). These studies showed that temporal averaging reduced the noise level from a control run in terms of certain meteorological variables such as zonal wind, precipitation, and ground temperature, which leads to the robustness of the climate change signal and enhances its detectability.

The spatial averaging operation is used explicitly or implicitly in most climate change impact studies when analyzing domain-averaged climate data, and its effect on the robustness of the climate change signal has been investigated (Räisänen 2001; Kendon et al. 2008; McSweeney and Jones 2013). Because the spatial distributions of the meteorological variables are less homogeneous than the temporal distribution, the signal is not monotonically enhanced by increasing the spatial scale. For example, over Alpine regions, a spatial averaging operation reduces the signal-to-noise ratio (SNR) of extreme precipitation and leads to difficulty in detecting its changing signal due to the complex terrain (Kendon et al. 2008). However, on average, the robustness of the climate change signal is considered to be enhanced by increasing the spatial averaging scales (Räisänen 2001).

Another important type of averaging operation used in climate change impact studies is ensemble averaging across multiple samples from a climatologically identical population (Brankovic and Palmer 1997). However, analyses for ensemble averaging are not necessary in the present study because its effects are independent of those of the temporal and spatial averaging operations, and it is beyond the scope of this study.

Although previous work has shown the effect of temporal and spatial averaging operations separately, their interrelated effect has not been investigated. The present study aims to reveal the relation between them because the relative magnitude of their effects is crucial for climate change impact assessments using various temporal and spatial scales or resolutions.

Each meteorological phenomenon has intrinsic temporal and spatial scales (Cushman-Roisin and Beckers 2011) and their variability obscures the climate change signal. The phenomena whose temporal and spatial scales are smaller than each averaging scale are smoothed out and the robustness of the signal is enhanced by the extent of the variability reduction. Therefore, the distribution of the temporal and spatial scales of the meteorological phenomena relating to precipitation events determines the effect of the averaging operation with the given averaging scales.

Even though both temporal and spatial averaging operations are considered to have the effect of enhancing the detectability of the climate change signal, researchers or end users in climate change assessments demand as high as possible resolution in both temporal and spatial directions; the smallest averaging scales are demanded. Therefore, there exists a trade-off relation between the two averaging scales given a required detectability level, e.g., SNR. Such a trade-off relation, if obtained quantitatively, is useful for researchers aiming to perform climate change assessments with a given set of climate simulations. When such researchers need to reduce the temporal averaging scales for some reason, they find that detection of climate change signals becomes difficult without additional experiments. In such cases, they can detect the climate change signal with the same robustness by increasing the spatial averaging scale. The necessary increase in the spatial scales is estimated using the trade-off relation. The trade-off relation can be used in an opposite situation, which requires the spatial averaging to be reduced.

The target variable in this study is precipitation because its change is of great concern in agriculture, ecology, and other interdisciplinary fields. In addition, choosing appropriate temporal and spatial averaging scales for precipitation change assessments is a challenging and important task because its change signal detection is more difficult than that of other meteorological variables like surface temperature. We analyze daily precipitation data from an atmospheric general circulation model (AGCM) that reflect convection structures and meteorological dynamics from the meso scale to the planetary scale (e.g., Madden-Julian Oscillation; MJO). The relation between the two averaging scales for precipitation data at all longitudes and seasons is calculated, and we seek its general characteristics in three lati- 

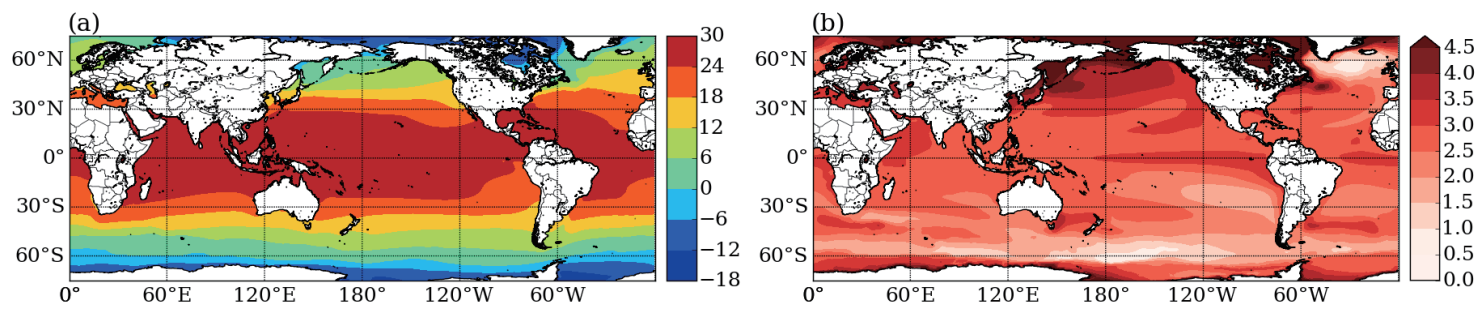

Fig. 1. Distributions of (a) mean present (1979-2003) sea surface temperature (SST) $\left({ }^{\circ} \mathrm{C}\right)$ from Hadley Centre's HadISST dataset and (b) mean future (2075-2099) SST change $\left({ }^{\circ} \mathrm{C}\right)$ from the present values (projected using the CMIP5 dataset). Both present and future SST fields are used as the lower boundary conditions of the atmospheric general circulation models in the present study.

tudinal regions: the tropics, the mid-latitudes, and the subpolar regions, because the meteorological dynamics in each region differ qualitatively and are expected to influence the detectability of the precipitation change signal.

The analyzed climate dataset and the procedure to obtain the relation between the temporal and spatial averaging scales are explained in Section 2. In Section 3 , we describe the trade-off relation between the two averaging scales. The well-known structures of the precipitation power spectrum related to the characteristics of the trade-off relation are also shown. In Section 4, we conclude our study and propose the usage of the trade-off relation for researchers conducting climate change impact assessments.

\section{Methodology}

\subsection{Dataset}

In order to compare present and future daily precipitation and detect its changing signal, the dataset from an AGCM is used. We used the latest $60-\mathrm{km}$ Meteorological Research Institute AGCM (MRI-AGCM3.2) (Mizuta et al. 2012). The model's convection scheme, which is important for the simulation of precipitation, is the Yoshimura scheme (Yoshimura et al. 2015).

For the present climate experiment, the observed inter-annually varying monthly-mean sea surface temperature (SST) and sea-ice concentration from the Hadley Centre's HadISST dataset (Rayner et al. 2003) are used to construct the lower boundary condition of the AGCM. Mean SST distribution is shown in Fig. 1a. On the other hand, the SST for the future experiment, which is shown as the mean change from the present experiment in Fig. 1b, is obtained by superimposing the trend and future changes in the Coupled Model Intercomparison Project Phase 5 (CMIP5) multi-model ensemble mean (Mizuta et al. 2014) on the de-trended observational SST explained above (Kitoh et al. 2015). The future SST distribution obtained by this method retains the observed inter-annual variability and the spatial patterns that are more likely to be included in observational data than in model data. The similarity between characteristics of the present and future SST distributions (except for their obvious increased signal), as shown in Fig. 1b, are necessary for the analysis of precipitation change because of the strong dependence of precipitation on the SST field. The emission scenario for the future experiment is the highest emissions scenario RCP8.5 (Moss et al. 2010). The other settings of the model we analyze can be found in a previous study (Mizuta et al. 2012).

The period of experiment integration is 1979-2003 for the present climate and 2075-2099 for the future climate. Because the averaging operation in the present study is a running average, the temporal averaging operation cannot be performed due to a lack of data on the first several days of the first year and the last several days of the last year. Therefore, the first and last years are eliminated from the analysis; the period for the analysis is thus 23 years and is hereafter referred to as $N_{\text {year }}$.

\subsection{Detection of climate change signal}

In the present study, we assume that the daily precipitation data samples from each year are mutually independent; thus, their spatio-temporal distribution in the present period $P_{p}$ can be expressed by the sample mean $\bar{P}_{p}$ and its deviation $P_{p}^{\prime}$; then

$$
\begin{aligned}
& P_{p}(x, y, d, i)=\bar{P}_{p}(x, y, d)+P_{p}^{\prime}(x, y, d, i), \\
& \bar{P}_{p}(x, y, d)=\frac{1}{N_{\text {year }}} \sum_{i=1}^{N_{\text {year }}} P_{p}(x, y, d, i),
\end{aligned}
$$

where $x, y, d$, and $i$ are longitude, latitude, day, and 
year, respectively, and the overline indicates mean values of samples from each year. Similarly, future daily precipitation $P_{f}$ is expressed as

$$
\begin{aligned}
& P_{f}(x, y, d, i)=\bar{P}_{f}(x, y, d)+P_{f}^{\prime}(x, y, d, i) \\
& \quad=\bar{P}_{p}(x, y, d)+\tilde{P}_{f}(x, y, d)+P_{f}^{\prime}(x, y, d, i),
\end{aligned}
$$

where $\tilde{P}_{f}$ is the forced component of future precipitation due to atmospheric composition change. The deviations of daily precipitation from each sample mean $P_{p}^{\prime}(x, y, d, i)$ and $P_{f}^{\prime}(x, y, d, i)$ can be regarded as components of internal variability. Note that internal variability consists of meteorological phenomena at all temporal and spatial scales, e.g., baroclinic waves in the mid-latitudes, equatorial waves in the tropics, and even the El Niño Southern Oscillation (ENSO) as an inter-annual variability.

The robustness of the precipitation change signal is estimated by the SNR as follows:

$$
\begin{aligned}
& S N R=\frac{\bar{P}_{f}(x, y, d)-\bar{P}_{p}(x, y, d)}{\sqrt{\left(\sigma_{p}^{2}+\sigma_{f}^{2}\right) / 2}}=\frac{\tilde{P}_{f}(x, y, d)}{\sigma_{S N R}}, \\
& \sigma_{p}^{2}=\overline{\left(P_{p}(x, y, t, d, i)-\bar{P}_{p}(x, y, t, d)\right)^{2}}, \\
& \sigma_{f}^{2}=\overline{\left(P_{f}(x, y, t, d, i)-\bar{P}_{f}(x, y, t, d)\right)^{2}},
\end{aligned}
$$

where $\sigma_{p}$ and $\sigma_{f}$ are the present and future standard deviations from each sample mean, respectively, and $\sigma_{S N R}$ indicates their mean. Although values of the SNR can take both positive and negative signs, the higher the absolute value of the SNR is, the more robust is the change signal and the more feasible is its detection.

In many climate change impact assessments, Student's $t$-tests (Hogg and Tanis 1977) are performed using $t=\sqrt{n / 2} S N R$, where $n$ is the number of sample members, and the statistical significance of a change signal of the sample mean values is examined. Because the probability distribution function (PDF) of precipitation with small averaging scales is distinctively different from the normal distribution (shown later), the Student's $t$-tests cannot be adopted to estimate significance levels of the precipitation change signal except when averaging scales are large enough and the central limit theorem can be applied. However, the magnitude of SNR as a function of the temporal and spatial averaging scales enables estimation of signal robustness since the SNR is identical to the $t$-value except for the coefficient of $\sqrt{n / 2}$.

\subsection{Averaging operation}

The daily precipitation data at a given location on a given day are averaged using the running average operation with temporal and spatial scales of $\left(L_{x y}, L_{t}\right)$ before calculating the SNR. The average precipitation $\hat{P}$ is calculated as follows:

$$
\hat{P}\left(x, y, t ; L_{x y}, L_{t}\right)=\left(\frac{1}{L_{x y}}\right)^{2} \frac{1}{L_{t}} \iiint P(x, y, t) \mathrm{d} t \mathrm{~d} y \mathrm{~d} x,(4)
$$

where, the variable of time is represented by $t$ whose units are days in the $N_{\text {year }}$ years consecutive time series. The geometric domains for the spatial averaging have the same length in both latitude and longitude. Because the precipitation data we analyzed are on lat/lon grids, sample weights for the integration, along with the meridional direction, are according to the area of the grid boxes, i.e., cosine of the latitude. The intervals of the integration for $x, y$, and $t$ are $[x-$ $\left.L_{x y} / 2, x+L_{x y} / 2\right],\left[y-L_{x y} / 2, y+L_{x y} / 2\right]$, and $\left[t-L_{t} / 2, t+\right.$ $\left.L_{t} / 2\right]$, respectively. By using Eq. (4), daily precipitation data of both present and future climates are transformed to the mean precipitation with the averaging scales $\left(L_{x y}, L_{t}\right)$ and then substituted into the procedure shown by Eqs. (1)-(3) to detect the precipitation change signal. The dependence of the SNR on the temporal and spatial averaging scales can be obtained and analyzed.

In the present study, we focus on daily to seasonal (91 days) temporal scales and on spatial scales from one-grid (approximately $60 \mathrm{~km}$ ) to a $3333-\mathrm{km}$ grid, which corresponds to $30^{\circ}$ at the Equator. Because the SNR dependence on the temporal and spatial averaging scales is expected to be different depending on season and location, maximal values of the two averaging scales are deliberately limited to moderate values in order to avoid mixing of data from different seasons and latitudinal regions. Instead of the limitation, we average the SNR dependence over all days in each year, over all longitudes, and over the latitudes ranging from $y_{\text {target }}-5^{\circ}$ to $y_{\text {target }}+5^{\circ}$ (where $y_{\text {target }}$ is a given latitude for the analysis), to obtain the SNR general characteristics regarding the two averaging scales, which provides a useful guideline for climate change impact assessments. As a result, the SNR values are reduced to a function of the temporal and spatial averaging scales $\left(L_{x y}, L_{t}\right)$ and the target latitude $y_{\text {target }}$. We call this operation "SNR averaging" to avoid confusion with the temporal and spatial averaging shown in Eq. (4). To clarify the difference between the tropics, the mid-latitudes, and the subpolar regions, the target latitudes are $65^{\circ} \mathrm{S}, 40^{\circ} \mathrm{S}$, 
the Equator, $40^{\circ} \mathrm{N}$, and $65^{\circ} \mathrm{N}$.

The sign of the precipitation change differs depending on location and season. In order to not cancel each other out in SNR averaging, the SNR values are normalized before the averaging by their own sign, which is obtained with maximal $L_{x y}$ and $L_{t}$. Therefore, the SNR values hereafter indicate the magnitude of the climate change signal, and do not include the sign.

\subsection{Spatio-temporal scales of precipitation phenomena}

The actual calculation of Eq. (4) is performed using the Fourier transform of the daily precipitation data. Both present and future daily precipitation as a function of longitude, latitude, and time $P(x, y, t)$ are transformed into Fourier space as $\mathcal{F}[P]\left(k_{x}, k_{y}, f\right)$, where $k_{x}$ is the zonal wavenumber, $k_{y}$ the meridional wavenumber, and $f$ the frequency. Performing the running average in physical space, as shown in Eq. (4), corresponds to multiplying the Fourier transform by cardinal sine functions in the Fourier space for each of the three dimensions, as follows (Smith 1997):

$$
\begin{aligned}
\widehat{\mathcal{F}} & P]\left(k_{x}, k_{y}, f ; L_{x y}, L_{t}\right) \\
= & \frac{\sin \left(M_{x} k_{x} / 2\right)}{M_{x} \sin \left(k_{x} / 2\right)} \times \frac{\sin \left(M_{y} k_{y} / 2\right)}{M_{y} \sin \left(k_{y} / 2\right)} \\
& \times \frac{\sin \left(M_{t} f / 2\right)}{M_{t} \sin (f / 2)} \times \mathcal{F}[P]\left(k_{x}, k_{y}, f\right),
\end{aligned}
$$

where $M_{x} M_{y}$, and $M_{t}$ indicate the number of gridpoint data used for the running average of $x, y$, and $t$, which are determined by $L_{x y}$ and $L_{t}$. Finally, mean precipitation $\hat{P}\left(x, y, t ; L_{x y}, L_{t}\right)$ is obtained by calculating the inverse Fourier transform of $\widehat{\mathcal{F}}[P]\left(k_{x}, k_{y}, f\right.$; $\left.L_{x y}, L_{t}\right)$. This procedure is much faster in computational time than calculating the integration in Eq. (4).

The domain of $P(x, y, t)$ consists of all longitudes for $x$, all days over 25 years for $t$, and from $y_{\text {target }}-$ $20^{\circ}$ to $y_{\text {target }}+20^{\circ}$ for $y$. After the inverse Fourier transform, SNR averaging is performed using the results of all longitudes, all days of $N_{\text {year }}$ years, and the meridional domain from $y_{\text {target }}-5^{\circ}$ to $y_{\text {target }}+5^{\circ}$. Since the maximal temporal and spatial averaging scales are 91 days and $30^{\circ}$, respectively, non-periodicity, along with time and the meridional direction, is not important. This series of calculations is performed for each set of $L_{t}$ and $L_{x y}$; consequently, the SNR, as a function of the temporal and spatial averaging scales and the target latitude, is obtained.

The procedure to calculate the averaged precip- itation using Fourier transform implies a relation between the SNR dependence on temporal and spatial averaging scales and the precipitation power spectrum representing spatio-temporal scales of precipitation-related meteorological phenomena. According to the Parseval theorem, the sum of the power spectrum (squared absolute values of Fourier transform) equals the variances of internal variability (hereafter called $\sigma_{\text {power }}^{2}$ ), which is expressed in the following discrete form:

$$
\begin{aligned}
& \sigma_{\text {power }}^{2}\left(L_{x y}, L_{t}, y_{\text {target }}\right) \\
& \equiv \frac{1}{N_{t} N_{x} N_{y}} \sum_{n=1}^{N_{t}} \sum_{m=1}^{N_{x}} \sum_{l=1}^{N_{y}} \mid \hat{P}_{n m l}\left(L_{x y}, L_{t}, y_{\text {target }}\right) \\
& -\left.\overline{\hat{P}}_{n m l}\left(L_{x y}, L_{t}, y_{\text {target }}\right)\right|^{2} \\
& =\frac{1}{\left(N_{t} N_{x} N_{y}\right)^{2}} \sum_{i=1}^{N_{t}} \sum_{j=1}^{N_{x}} \sum_{k=1}^{N_{y}}\left|\widehat{\mathcal{F}}[P]_{i j k}\left(L_{x y}, L_{t}, y_{\text {target }}\right)\right|^{2},
\end{aligned}
$$

where, $n, m$, and $l$ are the indices of time, longitude, and latitude, respectively; $i, j$, and $k$ are the indices of frequency, zonal wavenumber, and meridional wavenumber, respectively; and $N_{x}, N_{y}$, and $N_{t}$ are the total numbers of grid-point data along each direction. Performing SNR averaging on the variance $\sigma_{p}^{2}$ or $\sigma_{f}^{2}$, which constitutes $\sigma_{S N R}$, yields the mean variance as a function of temporal and spatial averaging scales and a given target latitude. This equals $\sigma_{\text {power }}^{2}$, for which either present or future precipitation is used in Eq. (6). The standard deviation $\sigma_{S N R}$ is the only term in Eq. (3) affected by temporal and spatial averaging because the numerator is a linear term and does not change due to the cancellation between the temporal and spatial averaging and SNR averaging. As a result, the dependence of the SNR on the two averaging scales derives from that of the standard deviation $\sigma_{S N R}$ and $\sigma_{\text {power }}^{2}$ and is related to the precipitation power spectrum.

The running average used for temporal and spatial averaging is categorized as a low-pass filtering operation. The extent to which the averaging operation with a given averaging scale attenuates the variability of precipitation is determined by the distribution of the Fourier transform of precipitation $\mathcal{F}[P]\left(k_{x}, k_{y}, f\right)$ or its power spectrum distribution. For example, the total variability of precipitation is largely decreased if its power spectrum has large amplitude in the frequency range that is eliminated by a temporal low-pass filter. The averaging operations decrease the standard deviation $\sigma_{S N R}$ representing the magnitude of the internal variability and consequently increase the SNR. A 


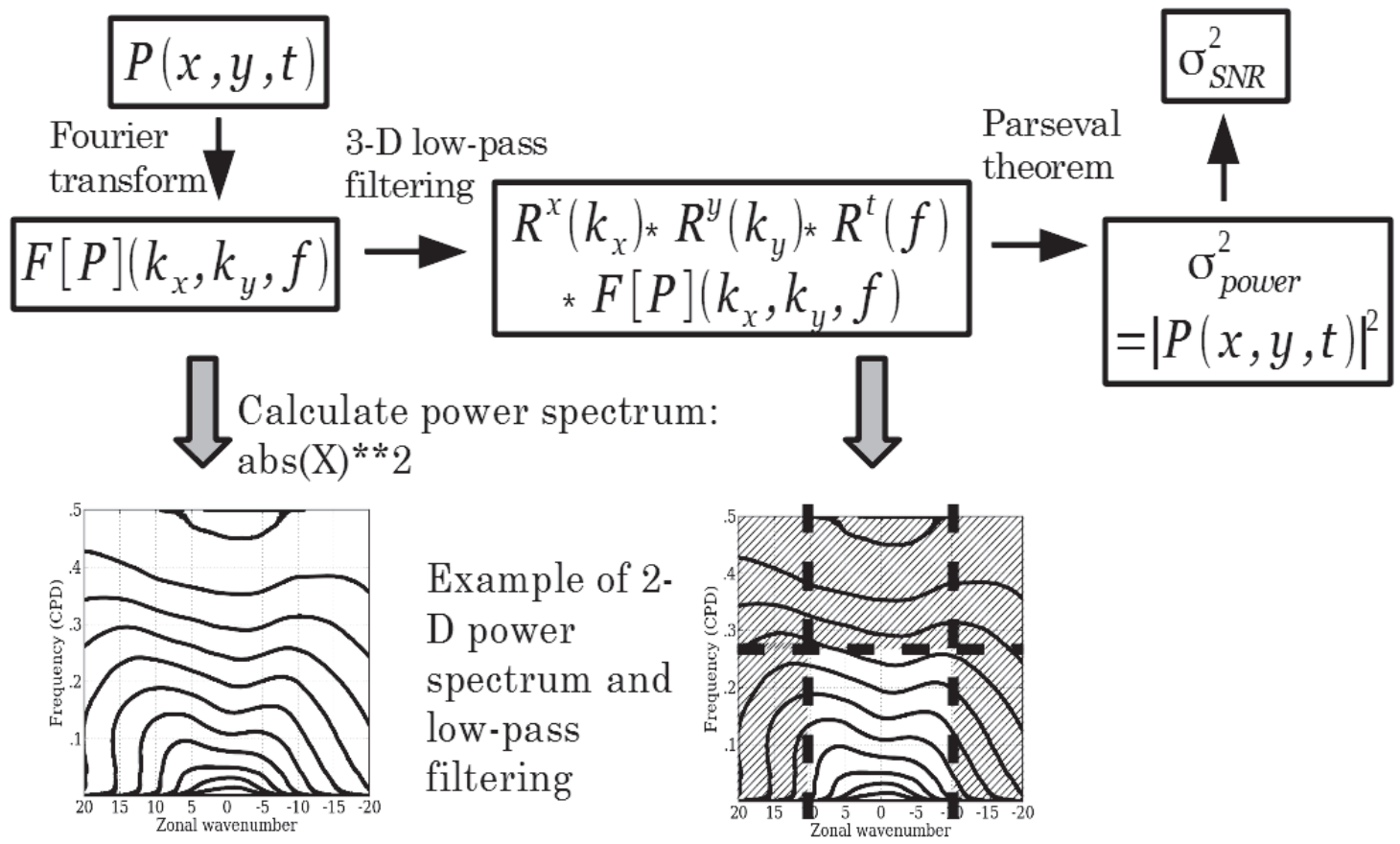

Fig. 2. Flowchart to obtain the standard deviation in the definition of the signal to noise ratio, which shows the relation between the structure of the power spectrum and the standard deviation. $R^{x}, R^{y}$, and $R^{t}$ are response functions for $x, y$, and $t$ (cardinal sine function), respectively, corresponding to the running average shown in (5).

schematic of the low-pass filtering operation and the precipitation power spectrum is shown in Fig. 2.

The precipitation power spectrum reflects rainfall systems and differs depending on factors such as location, season, and underlying surface (e.g., whether over continent or ocean), but its general features depend on latitude. In order to compare the characteristics of the power spectrum in the present study with those shown in previous studies (Wheeler and Kiladis 1999; Takayabu 1994a,b; Dell'Aquila et al. 2005), the two-dimensional (2-D) power spectrum summed with the meridional direction is shown. In addition, one-dimensional (1-D) power spectra, along with frequency, zonal wavenumber, and meridional wavenumber, are calculated to reveal the individual effect of the averaging operation, along with time, longitude, and latitude, respectively. If the characteristics of the power spectrum obtained from the dataset that we used are consistent with those of well-understood previous studies, the subsequent results of the standard deviation $\sigma_{S N R}$ and the SNR are justified and demonstrate the reliability of the trade-off relation between temporal and spatial averaging scales on the robustness of the precipitation change signal.

\section{Results}

\subsection{Effect of averaging operation}

First, we show some examples of the effect of the temporal and spatial averaging operations on the robustness of the precipitation change signal. The PDFs of daily precipitation at the Equator, $180^{\circ}$ in June-July-August (JJA) are indicated in Figs. 3a-d as an example for the tropics, while Figs. $3 \mathrm{e}-\mathrm{h}$ and $3 \mathrm{i}-1$ show those at $40^{\circ} \mathrm{N}$ and $65^{\circ} \mathrm{N}$ as examples in the mid-latitudes and the subpolar regions, respectively, and whose longitude and season are the same as those in Figs. 3a-d. Figures 3a, 3e, and 3i show local daily precipitation at a single grid point; Figs. $3 \mathrm{~b}, \mathrm{f}$, and $\mathrm{j}$ show the seasonally averaged precipitation $\left(L_{t}=91\right.$ days); Figs. 3c, $3 \mathrm{~g}$, and $3 \mathrm{k}$ show the domain-averaged precipitation $\left(L_{x y}=3333 \mathrm{~km}\right)$; and Figs. $3 \mathrm{~d}, 3 \mathrm{~h}$, and 31 show both seasonally and domain-averaged precipitation. All samples for obtaining the PDF were gathered from the whole averaging domain and JJA period for each figure. The robustness of the signal of precipitation increase is enhanced by the averaging operations due to the decreased sample variances (shown by standard deviations in each figure), and the change signals of both temporally and spatially 

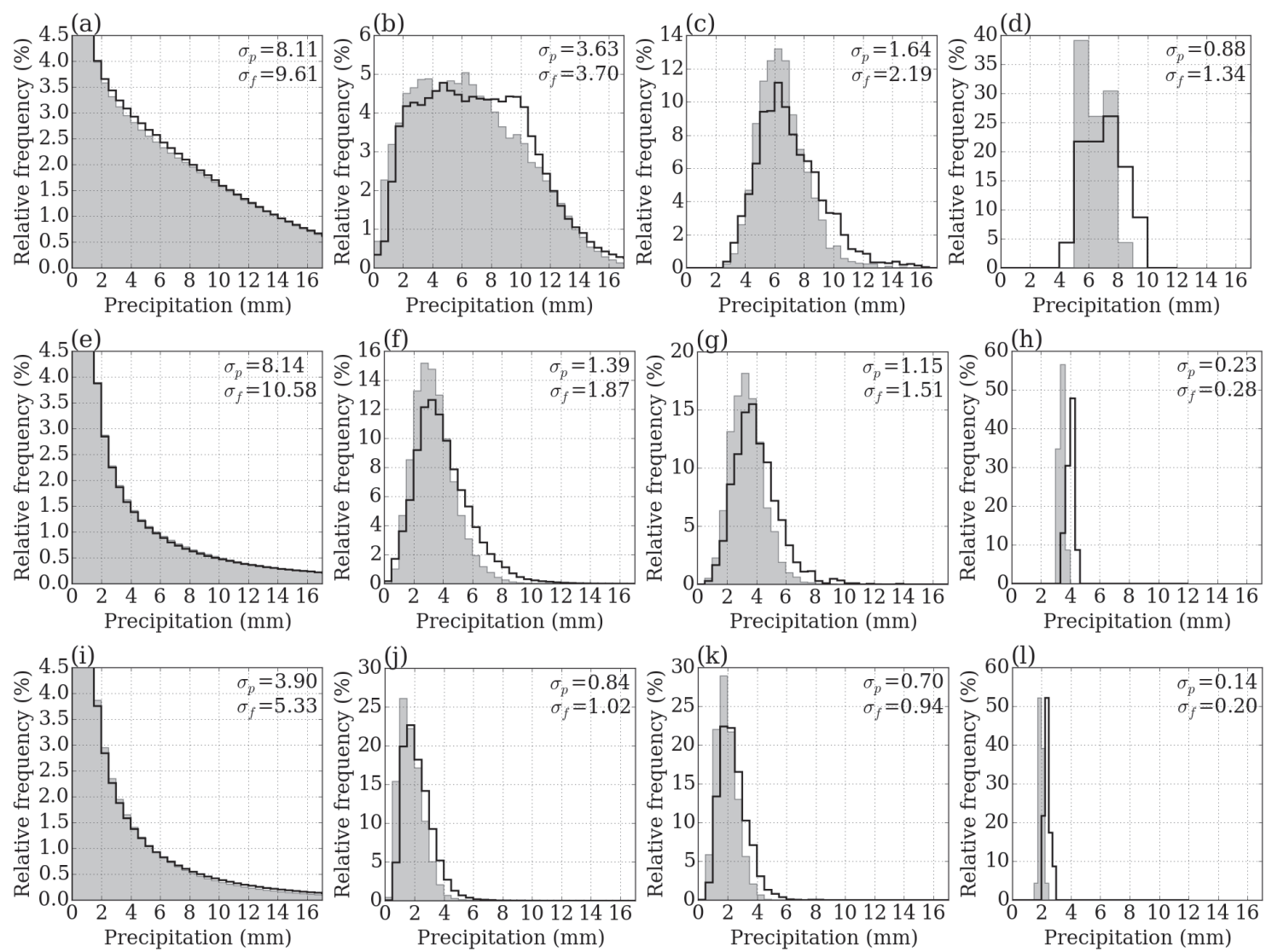

Fig. 3. (a-d) Relative frequency of (a) daily precipitation, (b) temporally averaged precipitation, (c) spatially averaged precipitation, and (d) tempo-spatially averaged precipitation at the Equator, $180^{\circ}$ in June-July-August (JJA). Present precipitation is shown by gray shading and future precipitation by a black line. The mean values $m_{p}$ (present) and $m_{f}$ (future) and standard deviations $\sigma_{p}$ (present) and $\sigma_{f}$ (future), together with the temporal and spatial averaging scales, are shown in the right upper corner of the panel. (e-h) Same as (a-d), but for $40^{\circ} \mathrm{N}$. (i-1) Same as (ad), but for $65^{\circ} \mathrm{N}$.

averaged precipitation values (Figs. 3d, h, 1) are most clear in all three latitudinal regions. In the tropics, the temporal averaging generates a smaller effect for reducing the sample variances than the spatial averaging, although both averaging operations cause decreases in the variances to approximately the same degree in the mid-latitudes and subpolar regions. This difference will be quantitatively shown and examined later.

Figure 4 shows an example of the JJA distribution of the SNR, which is obtained by Eq. (4) using the maximal values of $L_{x y}(3333 \mathrm{~km})$ and $L_{t}(91$ days) and a time $t$ that is set to 16 th July. The maximal grid-box size of $L_{x y}$ is shaded in Fig. 4 as an example of when the target latitude and longitude are at the Equator, $180^{\circ}$. The three locations where latitude and longi- tude are (the Equator, $\left.180^{\circ}\right),\left(40^{\circ} \mathrm{N}, 180^{\circ}\right)$, and $\left(65^{\circ} \mathrm{N}\right.$, $180^{\circ}$ ) have positive SNR values, which is consistent with the results in Figs. 3d, h, and 1.

The magnitude of the SNR value in Eq. (3) shows the robustness of the precipitation change signal. Figure 5 shows SNR distributions in terms of $L_{t}$ and $L_{x y}$ at the target latitudes: (a) the Equator, as being representative of the tropics; (b) $40^{\circ} \mathrm{S}$ and (c) $40^{\circ} \mathrm{N}$ for the mid-latitudes; and (d) $65^{\circ} \mathrm{S}$ and (e) $65^{\circ} \mathrm{N}$ for the subpolar regions. The units for the temporal and spatial averaging scales are days and $\mathrm{km}$, respectively. Comparison of the SNR among the three latitudinal regions indicates higher values in the subpolar regions of both the Northern and Southern Hemispheres (Figs. 5d, e). The high SNR values in the subpolar regions are attributed to the large percentage change 


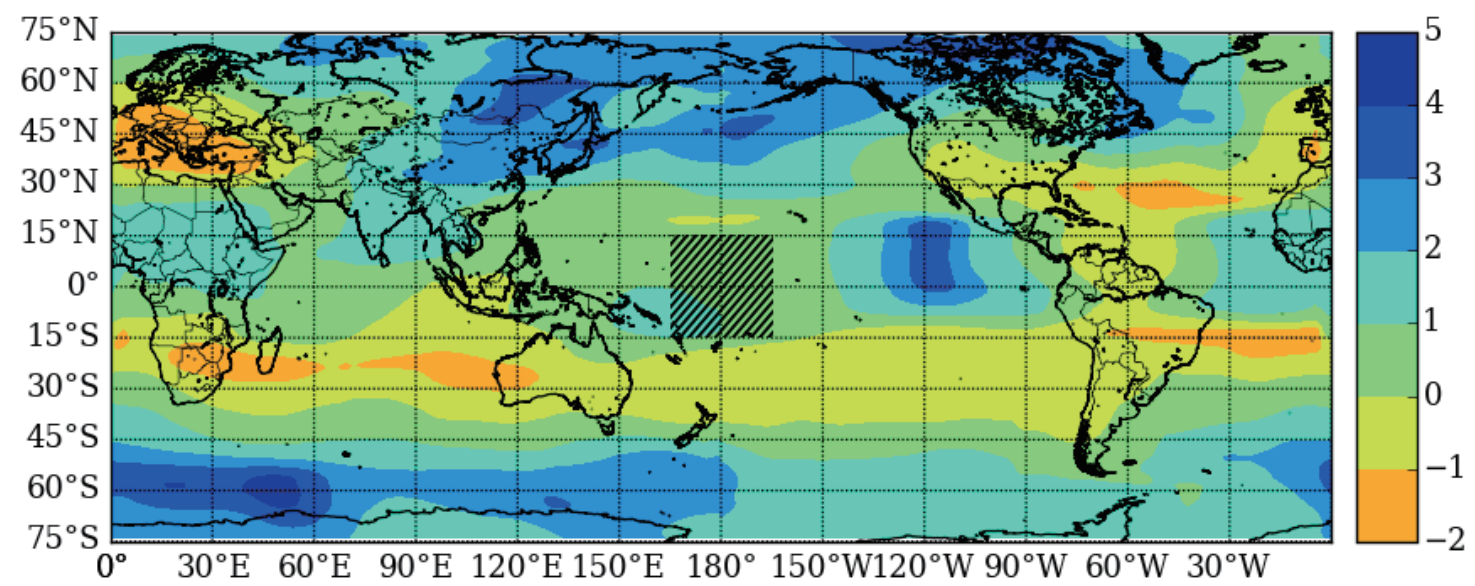

Fig. 4. The JJA global distribution of the SNR from the present climate to future one with maximal temporal and spatial averaging scales: $L_{t}=91$ days and $L_{x y}=3333 \mathrm{~km}$. The shaded domain centered at the Equator, $180^{\circ}$ indicates the domain size of the maximal spatial averaging scale.
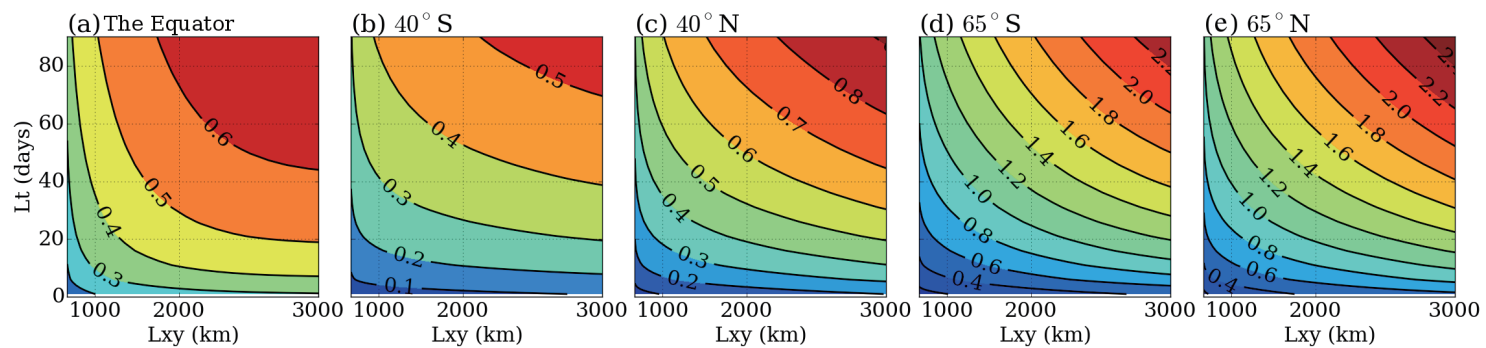

Fig. 5. The dependence of the SNR values on the temporal averaging scales $L_{t}$ and spatial averaging scales $L_{x y}$ at (a) the Equator, (b) $40^{\circ} \mathrm{S}$, (c) $40^{\circ} \mathrm{N}$, (d) $65^{\circ} \mathrm{S}$, and (e) $65^{\circ} \mathrm{N}$. The spatial averaging scales $L_{x y}$ are arranged in such a way that their squared values are linearly arranged on the $x$-axis. This enables the $x$-axis to be proportional to the number of grid-point data used to obtain the spatially averaged values.

of precipitation relative to the present climate, which appears in many future climate simulations, e.g., CMIP5 (Stocker et al. 2014). Figure 4 shows high SNR values in the subpolar regions as an example of the JJA period. In contrast, the values of SNR in the tropics and the mid-latitudes do not differ markedly from each other.

The SNR values in Fig. 5 increase monotonically with both temporal and spatial averaging scales, which is consistent with an intuitive understanding of the averaging operations in climate change assessments. The leftward tilted isolines of the SNR values indicate that the same SNR value is obtained by increasing the temporal scales when the spatial scales are decreased, and vice versa. Therefore, the isolines represent a trade-off relation between the temporal and spatial averaging scales. The SNR isolines in the tropics are more vertically tilted compared with those in the mid-latitudes and the subpolar regions. This difference indicates that in the tropics, the temporal averaging operation has a smaller effect on detecting the precipitation change signal than the spatial averaging operation, which is also apparent in the example shown in Fig. 3.

In order to highlight the characteristics of the trade-off relation, change ratios of the temporal and spatial averaging scales are examined when the temporal or spatial averaging scales are changed and the SNR value is maintained constant. Two change ratios $\alpha$ and $\beta$ satisfy a relation $\operatorname{SNR}\left(L_{x y}, L_{t}\right)$ $=\operatorname{SNR}\left(\alpha L_{x y}, \beta^{-1} L_{t}\right)$ and are schematically explained in Fig. 6a. Figure $6 \mathrm{~b}$ is a scatter diagram of the two change ratios $\alpha$ ( $x$-axis) and $\beta$ (y-axis) for the results in the tropics (Equator is red points), the mid-lati- 
(a)

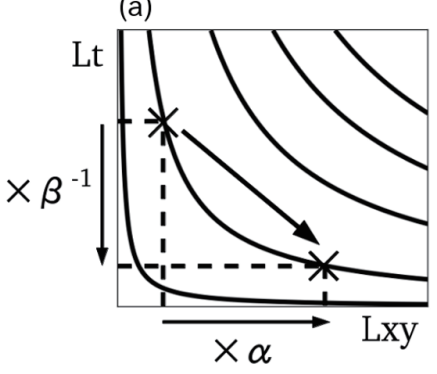

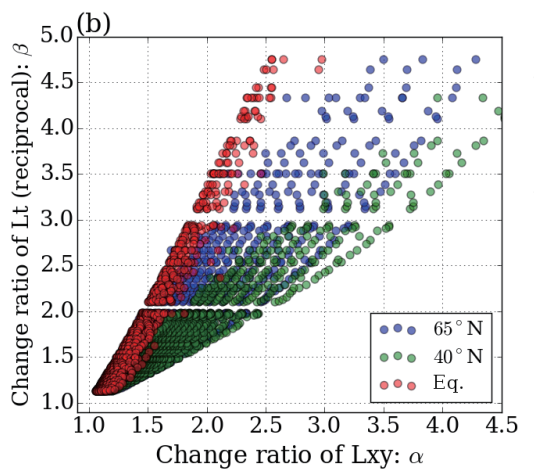

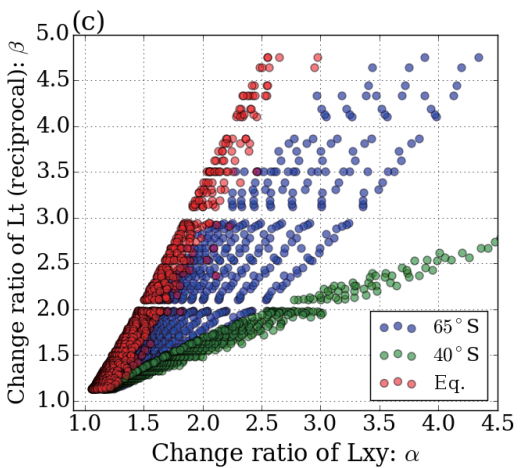

Fig. 6. (a) Schematic of the definition of change ratios of the spatial and temporal averaging scales $\alpha$ and $\beta$, respectively. Scatter diagrams showing the relation between the two change ratios corresponding to the trade-off relation shown in Fig. 5 for (b) the Southern Hemisphere and (c) the Northern Hemisphere. Blue, green, and red points represent the results for latitudes $65^{\circ} \mathrm{N}\left(65^{\circ} \mathrm{S}\right), 40^{\circ} \mathrm{N}\left(40^{\circ} \mathrm{S}\right)$, and the Equator, respectively. For comparison, the same result at the Equator is drawn in both (b) and (c).
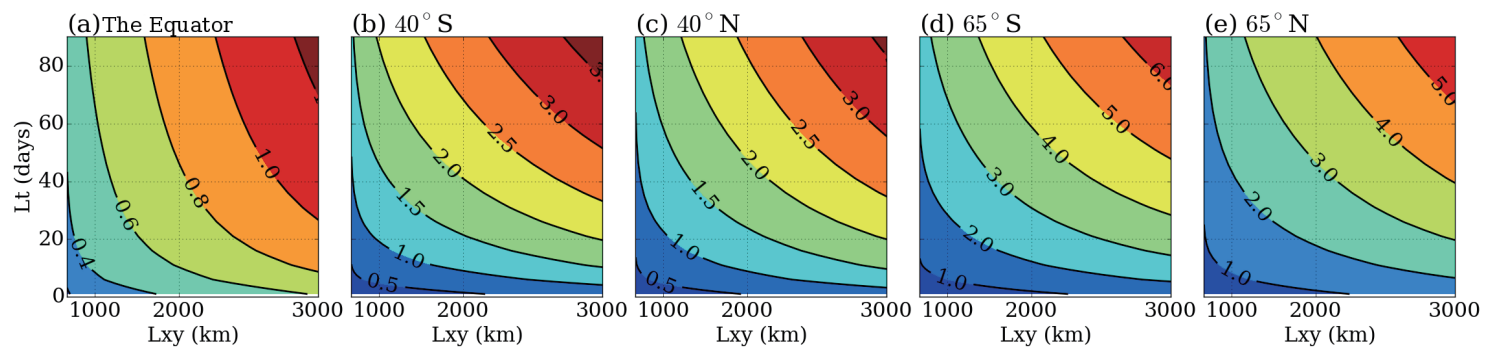

Fig. 7. Similar to Fig. 5, except that the variable is the reciprocal of the standard deviation $\sigma_{S N R}$.

tudes $\left(40^{\circ} \mathrm{N}\right.$ is green points), and the subpolar regions $\left(65^{\circ} \mathrm{N}\right.$ is blue points). The results for the Southern Hemisphere (Equator, $40^{\circ} \mathrm{S}$, and $65^{\circ} \mathrm{S}$ ) are indicated in Fig. 6c, with the identical results from the tropics shown for comparison. When drawing this scatter diagram, SNR values near both axes $\left(L_{x y} \leqq 625\right.$ $\mathrm{km}$ and $L_{t} \leqq 5$ days) were omitted because results obtained from these ranges were separated from the other results. However, the scatter diagram is considered to represent the general characteristics of SNR distribution because the omitted range is small compared with the overall range shown in Fig. 5.

The scatter points across the three latitudinal regions are separated, particularly in the Southern Hemisphere, and the tendency in the tropics for the temporal averaging operation to generate a smaller effect than the spatial averaging operation is reproduced. The trade-off relation at the subpolar regions is found to occupy an intermediate position between those at the tropics and mid-latitudes. The temporal averaging scales have to be increased approximately 4.5 times in the tropics when the spatial averaging scales are decreased 2.5 times, while only approximately $2\left(40^{\circ} \mathrm{S}\right)$ or $2.5\left(40^{\circ} \mathrm{N}\right)$ times increases of the temporal averaging scale are necessary in the mid-latitudes.

The results of the standard deviation $\sigma_{S N R}$ are shown to reveal the origin of the difference among the SNR distributions in the three latitudinal regions. The standard deviation is a function of the temporal and spatial averaging scales and the target latitude due to the same operation as SNR averaging. The distribution of the reciprocal of the standard deviation is shown in Fig. 7. The isolines for the tropics are tilted vertically compared with those for the mid-latitudes and the subpolar regions, which is consistent with the results of the SNR. As already explained in the methodology section, the dependence of the SNR on the averaging scales is derived from that of the standard deviation $\sigma_{S N R}$; thus, the differences among the three 


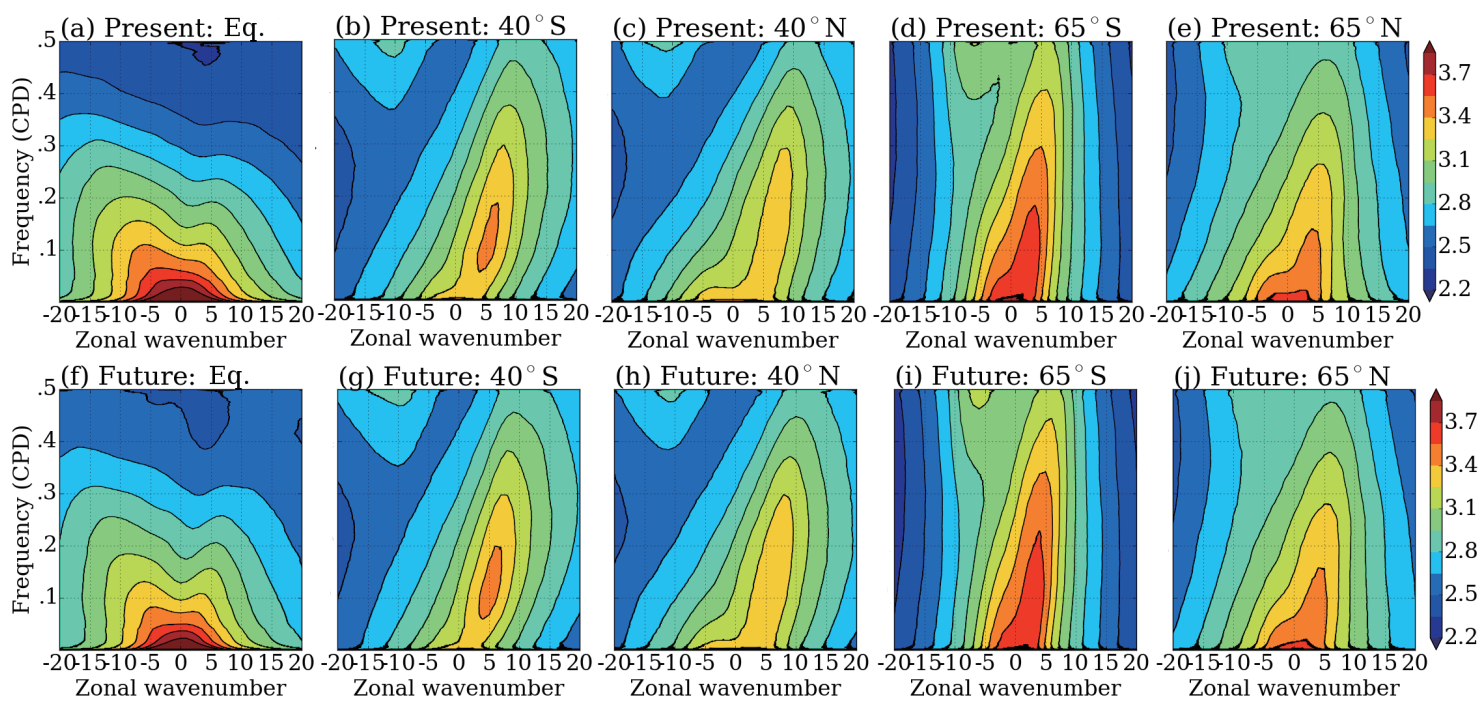

Fig. 8. Two dimensional precipitation power spectra for the present climate as a function of frequency and zonal wavenumber at (a) the Equator, (b) $40^{\circ} \mathrm{S}$, (c) $40^{\circ} \mathrm{N}$, (d) $65^{\circ} \mathrm{S}$, and (e) $65^{\circ} \mathrm{N}$. Those for the future climate are similarly shown in $(\mathrm{f}-\mathrm{j})$. The value of a power spectrum density is normalized (see text) and transformed by taking its common logarithm. The color bars and contours in all panels are identical.

latitudinal regions, as represented by the SNR isolines in Fig. 5, are attributed to the characteristics of the standard deviation $\sigma_{S N R}$ at each latitude.

\subsection{Precipitation power spectrum}

In this subsection, we show the results for the precipitation power spectrum which determine the characteristics of the trade-off relation. Figure 8 shows the 2-D power spectrum of the present precipitation $\left|\mathcal{F}^{y}[P]\left(k_{x}, f\right)\right|^{2}$, for which three-dimensional (3-D) power spectra are calculated using the precipitation data and summed along the meridional wavenumber at (a) the Equator, (b) $40^{\circ} \mathrm{S}$, (c) $40^{\circ} \mathrm{N}$, (d) $65^{\circ} \mathrm{S}$, and (e) $65^{\circ} \mathrm{N}$ for the present precipitation $P_{p}$. Similar results for the future precipitation $P_{f}$ are indicated in Figs. $8 \mathrm{f}-\mathrm{j}$. The unit of frequency is cycles per day (CPD) and the power spectrum is shown up to the maximum frequency of our daily data, i.e., 0.5 CPD. On the other hand, the high wavenumber range is omitted in order to highlight the energy-concentrated low wavenumber range and to compare with the results of previous studies (Wheeler and Kiladis 1999; Takayabu 1994a, b; Dell'Aquila et al. 2005). In order to obtain the general characteristics of the power spectrum, a running average operation along with frequency and a 1-2-1 filtering operation along with wavenumber (Wheeler and Kiladis 1999) are performed, and the smooth isolines are shown. The absolute values of the power spectrum are normalized in such a way that $\iint\left|\mathcal{F}^{y}[P]\left(k_{x}, f\right)\right|^{2} d k_{x} d f=1$, and are transformed by taking their common logarithm.

Compared with the tropics, a power spectrum "ridge" extends to the high frequency region in the mid-latitudes. Since a positive wavenumber indicates eastward moving waves, the spectral components of the ridge correspond to baroclinic waves generating rainfall derived from mid-latitude cyclones. Such a frequency-wavenumber structure has already been shown in previous studies (Dell'Aquila et al. 2005). The power spectrum in the subpolar regions exhibits similar eastward moving waves, but it is concentrated in the low frequency region to some extent. The moving waves are considered to be baroclinic waves, and the low-frequency spectral components may be related to long-term variations such as Arctic oscillation or Antarctic oscillation (Fyfe et al. 1999). However, the detailed identification of each spectral component in the subpolar regions is beyond the scope of the study. On the other hand, a large fraction of the power spectrum of precipitation in the tropics is concentrated in a low frequency region (showing like a red noise), which is an identical characteristic to that shown in previous studies (Wheeler and Kiladis 1999; Takayabu 1994a, b). The model we used reproduces well-understood meteorological structures like ENSO, MJOs, and the Intertropical Convergence 
Zone (Mizuta et al. 2012), and these are included as low-frequency structures. According to a previous study (Wheeler and Kiladis 1999), the Kelvin wave, which is one of the equatorial waves in the tropics, is embedded in the power spectrum; this is represented as a small ridge in the eastward moving region in Figs. 8a and 8f. The characteristics shown in the power spectrum results are well accepted in the meteorological community and qualitatively well reproduced in the AGCM. The results of the future precipitation power spectrum have the same features as those of the present precipitation.

In order to reveal the relation between the structure of the power spectrum and the distribution of the SNR in detail, 1-D power spectra as functions of (a) and (b) zonal wavenumber, (c) and (d) meridional wavenumber, and (e) and (f) frequency are shown in Fig. 9. The color of the lines indicates the latitudinal region (red is the Equator, green is $40^{\circ} \mathrm{S}$, and blue is $65^{\circ} \mathrm{S}$ ), and the solid and dashed lines show the results for the present and future climates, respectively. For clarity of the figure, the results from the Northern and Southern Hemispheres are separated (left and right columns, respectively) and results in the tropics are shown in both columns. All of the power spectra are calculated from the 3-D power spectrum and normalized and smoothed in the same manner as the 2-D power spectrum. The wavenumber in Figs. 9a-d is arranged on a logarithmic scale to show its entire range (not shown in Fig. 8), and the values of the power spectrum are multiplied by their wavenumber so that the area under the curve is proportional to the variances at the local wavenumber. The dashed black lines indicate the first wavenumber (Figs. 9a-d) and frequency (Figs. 9e-f) at which the amplitudes of the power spectrum are attenuated to zero when the temporal and spatial averaging scales are prescribed as each maximum value: 91 days and $3333 \mathrm{~km}$, respectively, although the cardinal sine function in Eq. (5) has multiple roots or zero points.

The effect of the temporal and spatial averaging operation can be estimated by the ratios of the power spectrum remaining after each low-pass filter along with wavenumber or frequency to the total power spectrum, e.g., the area to the left of the vertical dashed line in Fig. 9 when the averaging scales are set to maximal values. The ratio for the tropics in Figs. $9 \mathrm{a}$ and $9 \mathrm{~b}$ is found to be comparable to, or larger than, the other latitudinal regions, while that for the tropics in Figs. 9c and 9d is slightly smaller. Therefore, the total effect of spatial averaging in reducing the variability and enhancing the robustness of the precipitation change signal is approximately the same across all three latitudinal regions. On the other hand, in Figs. 9e and 9f, a relatively large amount of the power spectrum for the precipitation in the tropics is concentrated in the low-frequency region, whilst for the precipitation in the mid-latitudes, a relatively large amount of power spectrum is in the high-frequency region. The power spectrum of the subpolar regions takes an intermediate position between those of the tropics and the mid-latitudes. As a result, the effect of temporal averaging is smallest in the tropics, followed by that in the subpolar regions, due to the low ratio of the power spectrum cut off by the temporal low-pass filter.

A similar inference is valid when comparing the Northern and Southern Hemispheres. The large deviation of the results in the Southern Hemisphere from those in the tropics, which is shown for the trade-off relation (Fig. 6c), is attributed to the small concentration in the low-frequency region (green lines in Fig. 9f). Comparing the results from the present and future experiments, the qualitative difference among the three latitudinal regions is retained in spite of their individual changes, such as the rightward shift of the spatial power spectrum of the tropics (red lines in Figs. 9a-d), i.e., the scale of precipitation events becoming smaller. As a result, the qualitative features of the precipitation power spectrum are found to be consistent with those of the trade-off relation at each latitude.

\section{Discussion and conclusion}

The present study showed the dependence of the detectability of the precipitation change signal toward the end of the 21 st century on the temporal and spatial averaging scales and proposed a trade-off relation between them by analyzing the daily precipitation data from AGCM results. The general characteristics of the trade-off relation are obtained in the tropics, the mid-latitudes, and the subpolar regions. The trade-off relation differs qualitatively depending on latitude, and the effect of temporal averaging in the tropics is found to be relatively small compared to those at the mid-latitudes and subpolar regions.

Our analyses are performed using only model-simulated climate data, but not observational data. Although using observational precipitation data instead of those from the model may cause a quantitative change in the trade-off relation (as compared with our results), its qualitative characteristics are verified by analyzing the precipitation power spectrum. The distributions of the precipitation power 

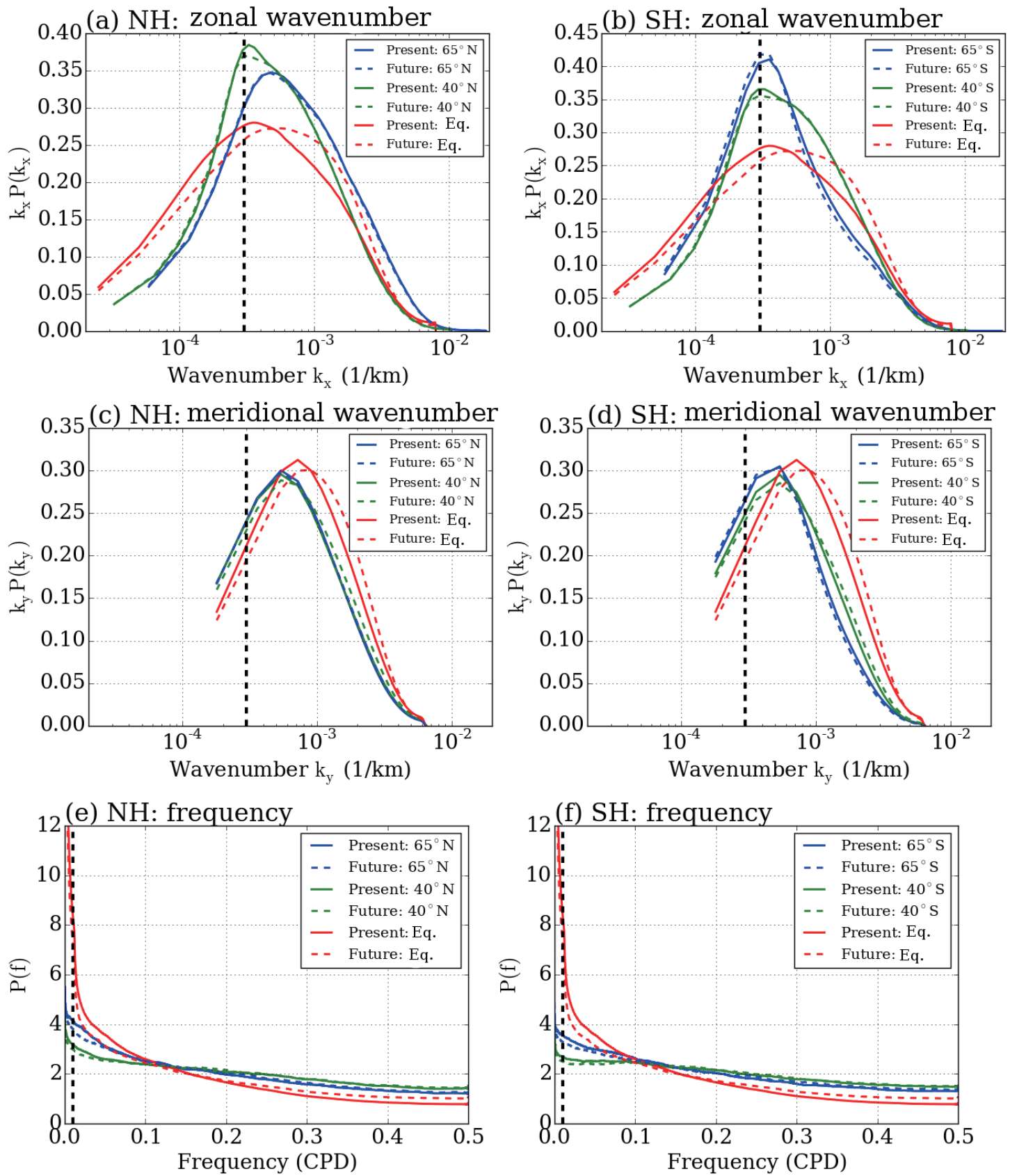

Fig. 9. (a, c, e) Power spectra of precipitation at (red lines) the Equator, (green) $40^{\circ} \mathrm{N}$, and (blue) $65^{\circ} \mathrm{N}$ in terms of (a) zonal wavenumber, (c) meridional wavenumber, and (e) frequency. The solid and dashed lines denote the present and future climates, respectively. The dashed thick line shows the first wavenumber (frequency) at which the amplitude of a power spectrum is attenuated to zero when the spatial (temporal) averaging scales is prescribed to be $3333 \mathrm{~km}$ (91 days) in Figs. 9a, c (Fig. 9e). (b, d, f) Same as (a, c, e), but for the Southern Hemisphere.

spectra in the three latitudinal regions are reproduced in a manner consistent with previous studies (Wheeler and Kiladis 1999; Takayabu 1994a, b; Dell'Aquila et al. 2005), and their differences between present and future climates are smaller than those among the three latitudinal regions. Therefore, the results for the 
trade-off relation and their differences among the latitudinal regions are guaranteed qualitatively because the precipitation power spectrum entirely determines the general features of the trade-off relation in each latitude.

The detectability of climate change signals has a strong relationship with the two averaging scales. If both averaging scales are decreased, the detectability may be lost; thus, the relationship is regarded as another trade-off relation. The trade-off relation between the two averaging scales we calculated in the present study is useful when either averaging scale is decreased and the signal robustness must be maintained; this relation provides information on how the other averaging scale needs to be increased. Such usefulness stands out when climate simulations are given (e.g., CMIP experiments) and the number of ensemble members cannot be increased.

Although climate change assessment researchers can use the trade-off relation we provided as a first estimation when determining the temporal and spatial averaging scales for their analyses, quantitative features of the trade-off relation can also depend on longitude, season, and underlying surface (e.g., whether continent or ocean). More detailed estimation of the trade-off relation for such conditions is more useful for local climate change assessments and is the subject of future study.

\section{Acknowledgments}

We are thankful to Dr. R. Mizuta and Dr. K. Yoshida of the Meteorological Research Institute for providing the results of the AGCMs analyzed in this paper. This research is supported by the SOUSEI program of MEXT, Japan.

\section{References}

Brankovic, Č., and T. N. Palmer, 1997: Atmospheric seasonal predictability and estimates of ensemble size. Mon. Wea. Rev., 125, 859-874.

Chervin, R. M., and S. H. Schneider, 1976: A study of the response of NCAR GCM climatological statistics to random perturbations: Estimating noise levels. $J$. Atmos. Sci., 33, 391-404.

Chervin, R. M., W. L. Gates, and S. H. Schneider, 1974: The effect of time averaging on the noise level of climatological statistics generated by atmospheric general circulation models. J. Atmos. Sci., 31, 2216-2219.

Cushman-Roisin, B., and J.-M. Beckers, 2011: Introduction to Geophysical FLuid Dynamics: Physical and Numerical Aspects, 2nd edition. Academic Press, 875 pp.

Dell'Aquila, A., V. Lucarini, P. M. Ruti, and S. Calmanti,
2005: Hayashi spectra of the northern hemisphere mid-latitude atmospheric variability in the NCEPNCAR and ECMWF reanalyses. Climate Dyn., 25, 639-652.

Fyfe, J. C., G. J. Boer, and G. M. Flato, 1999: The Arctic and Antarctic Oscillations and their projected changes under global warming. Geophys. Res. Lett., 26, 16011604.

Hogg, R. V., and E. A. Tanis, 1977: Probability and Statistical Inference. Vol. 993, Macmillan, New York, USA.

Kendon, E. J., D. P. Rowell, R. G. Jones, and E. Buonomo, 2008: Robustness of future changes in local precipitation extremes. J. Climate, 21, 4280-4297.

Kitoh, A., T. Ose, and I. Takayabu, 2015: Dynamical downscaling for climate projection with high-resolution MRI AGCM-RCM. J. Meteor. Soc. Japan, 94A, 1-16.

Leith, C. E., 1973: The standard error of time-average estimates of climatic means. J. Appl. Meteor., 12, 10661069.

McSweeney, C. F., and R. G. Jones, 2013: No consensus on consensus: The challenge of finding a universal approach to measuring and mapping ensemble consistency in GCM projections. Climate Change, 119, 617-629.

Mizuta, R., H. Yoshimura, H. Murakami, M. Matsueda, H. Endo, T. Ose, K. Kamiguchi, M. Hosaka, M. Sugi, S. Yukimoto, S. Kusunoki, and A. Kioh, 2012: Climate simulations using MRI-AGCM3. 2 with $20-\mathrm{km}$ grid. J. Meteor. Soc. Japan, 90, 233-258.

Mizuta, R., O. Arakawa, T. Ose, S. Kusunoki, H. Endo, and A. Kitoh, 2014: Classification of CMIP5 future climate responses by the tropical sea surface temperature changes. SOLA, 10, 167-171.

Moss, R. H., J. A. Edmonds, K. A. Hibbard, M. R. Manning, S. K. Rose, D. P. van Vuuren, T. R. Carter, S. Emori, M. Kainuma, T. Kram, G. A. Meehl, J. F. B. Mitchell, N. Nakicenovic, K. Riahi, S. J. Smith, R. J. Stouffer, A. M. Thomson, J. P. Weyant, and T. J. Wilbanks, 2010: The next generation of scenarios for climate change research and assessment. Nat., 463, 747-756.

Räisänen, J., 2001: $\mathrm{CO}_{2}$-induced climate change in CMIP2 experiments: Quantification of agreement and role of internal variability. J. Climate, 14, 2088-2104.

Rayner, N. A., D. E. Parker, E. B. Horton, C. K. Folland, L. V. Alexander, D. P. Rowell, E. C. Kent, and A. Kaplan, 2003: Global analyses of sea surface temperature, sea ice, and night marine air temperature since the late nineteenth century. J. Geophys. Res., 108, 4407, doi:10.1029/2002JD002670.

Smith, S. W., 1997: The Scientist and Engineer's Guide to Digital Signal Processing. California Technical Pub., San Diego, USA, 626 pp.

Stocker, T. F., D. Qin, G. K. Plattner, M. Tignor, S. K. Allen, J. Boschung, A. Nauels, Y. Xia, V. Bex, and P. M. Midgley, 2014: Climate Change 2013: The Physical Science Basis. Cambridge University Press 
Cambridge, UK and NY, 1535 pp.

Takayabu, Y. N., 1994a: Large-scale cloud disturbances associated with equatorial waves. Part I: Spectral features of the cloud disturbances. J. Meteor. Soc. Japan, 72, 433-448.

Takayabu, Y. N., 1994b: Large-scale cloud disturbances associated with equatorial waves. Part II: Westward-propagating inertio-gravity waves. J. Meteor. Soc. Japan, 72, 451-465.
Wheeler, M., and G. N. Kiladis, 1999: Convectively coupled equatorial waves: Analysis of clouds and temperature in the wavenumber-frequency domain. $J$. Atmos. Sci., 56, 374-399.

Yoshimura, H., R. Mizuta, and H. Murakami, 2015: A spectral cumulus parameterization scheme interpolating between two convective updrafts with semi-lagrangian calculation of transport by compensatory subsidence. Mon. Wea. Rev., 143, 597-621. 